\title{
A Functional Taxonomy of Logic Puzzles
}

Citation for published version (APA):

Hufkens, L. V., \& Browne, C. (2019). A Functional Taxonomy of Logic Puzzles. In D. Perez Liebana, \& S. Mostaghim (Eds.), IEEE Conference on Games 2019 (pp. 642-645). IEEE.

https://doi.org/10.1109/cig.2019.8848107

DOI:

10.1109/cig.2019.8848107

Document status and date:

Published: 26/09/2019

Document Version:

Publisher's PDF, also known as Version of record

Document license:

Unspecified

Please check the document version of this publication:

- A submitted manuscript is the version of the article upon submission and before peer-review. There can be important differences between the submitted version and the official published version of record. People interested in the research are advised to contact the author for the final version of the publication, or visit the DOI to the publisher's website.

- The final author version and the galley proof are versions of the publication after peer review.

- The final published version features the final layout of the paper including the volume, issue and page numbers.

Link to publication

\section{General rights}

Copyright and moral rights for the publications made accessible in the public portal are retained by the authors and/or other copyright owners and it is a condition of accessing publications that users recognise and abide by the legal requirements associated with these rights.

- Users may download and print one copy of any publication from the public portal for the purpose of private study or research.

- You may not further distribute the material or use it for any profit-making activity or commercial gain

- You may freely distribute the URL identifying the publication in the public portal.

If the publication is distributed under the terms of Article 25fa of the Dutch Copyright Act, indicated by the "Taverne" license above, please follow below link for the End User Agreement:

https://www.ou.nl/taverne-agreement

Take down policy

If you believe that this document breaches copyright please contact us at:

pure-support@ou.nl

providing details and we will investigate your claim.

Downloaded from https://research.ou.nl/ on date: 26 Apr. 2023 


\title{
A Functional Taxonomy of Logic Puzzles
}

\author{
Lianne V. Hufkens Cameron Browne \\ Department of Data Science and Knowledge Engineering \\ Maastricht University \\ Maastricht, The Netherlands \\ \{lianne.hufkens, cameron.browne\}@ maastrichtuniversity.nl
}

\begin{abstract}
There currently exists no taxonomy for the full range of puzzles including "pen \& paper" Japanese logic puzzles. We present a functional taxonomy for these puzzles in preparation for implementing them in digital form. This taxonomy reveals similarities and differences between these puzzles and locates them within the context of single player games.
\end{abstract}

Index Terms-games, puzzles, logic, taxonomy, classification

\section{INTRODUCTION}

This work is part of a project on digitally implementing the full range of pen $\&$ paper puzzles for computer analysis. In order to model these puzzles, it is useful to devise a taxonomy to highlight differences and similarities between them to facilitate their implementation.

We define a puzzle as a problem with defined steps for achieving one or more defined solutions, such that the challenge contains all information needed to achieve its own solution. Puzzles are distinct from games as they lack the adversarial element: "Puzzles are solved. Games are won.'11

We focus on the group of puzzles commonly known as Japanese logic puzzles [1], of which Sudoku (Fig. 11) is the most famous example. These puzzles typically have the following characteristics:

- single player,

- simple rules,

- language independent,

- solvable by deduction,

- monotonic accumulation of information, and

- single unique solution.

While there exist taxonomies for various puzzle types, such as mechanical puzzles ${ }^{2}$ we found no existing taxonomy for this class of puzzles and so performed the following analysis.

In particular, we aim to determine whether our target range of puzzles can be categorised by goal and process, and whether that produces a natural taxonomy that tallies with the user experience when solving these puzzles. This short paper describes our taxonomy of logic puzzles and how it fits into the larger taxonomy of puzzles in general.

\section{PRIOR WORK}

Tokyo-based publisher Nikoli is the world's foremost producer of pen \& paper logic puzzles. Our initial corpus of examples was therefore derived from the catalogue of Nikoli puzzle

${ }^{1}$ https://www.quora.com/What-is-the-difference-between-a-game-and-a-puzzle ${ }^{2}$ Mechanical puzzle taxonomies: http://www.robspuzzlepage.com/classif $2 . h t m$

978-1-7281-1884-0/19/\$31.00 @2019 European Union
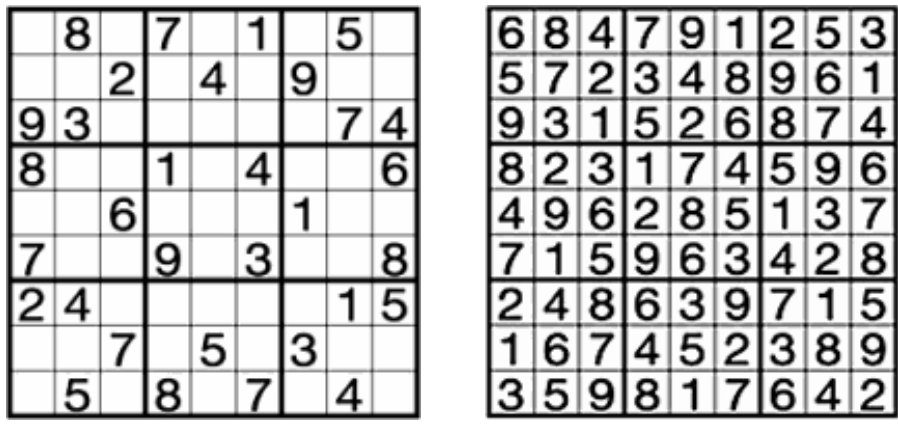

Fig. 1: A Sudoku challenge (left) and solution (right).

books, with further examples and information on Nikoli-style logic puzzles obtained from online sources including [2], [3], [4], [5] and [6].

Some simple schemes for logically grouping logic puzzles already exist. For example, our final taxonomy was inspired by the classification found at [2], and Nikoli [3] uses the following scheme:

- 数字 (numbers)

- 言葉 (language)

- 絵 (picture)

Another scheme used by online puzzle editor PUZ-PRE ${ }^{3}$ groups the available puzzles with some similarity to our categories, but without imposing any hierarchical structure nor defining their categories. However, these existing schemes are very broad and do not have any hierarchy. They are thus insufficient for our purposes, as they do not reveal similarities and differences between the puzzle groups with any depth.

According to Johnson: "There is no discipline of 'puzzle studies", the closest being the field of games study, so we apply similar game-based principles in our analysis [7]. Our classification scheme, based on 100 puzzles from the aforementioned sources, identifies them by:

1) Play mechanism: What the solver writes or draws.

2) Goal: What the player aims to achieve.

Puzzles of the same category in our taxonomy share rule characteristics and generally look visually similar.

\section{TAXONOMY}

Fig. 2 shows our taxonomy of logic puzzles, and how it fits into the broader context of single player games. We distinguish

${ }^{3}$ PUZ-PRE url: http://pzv.jp 


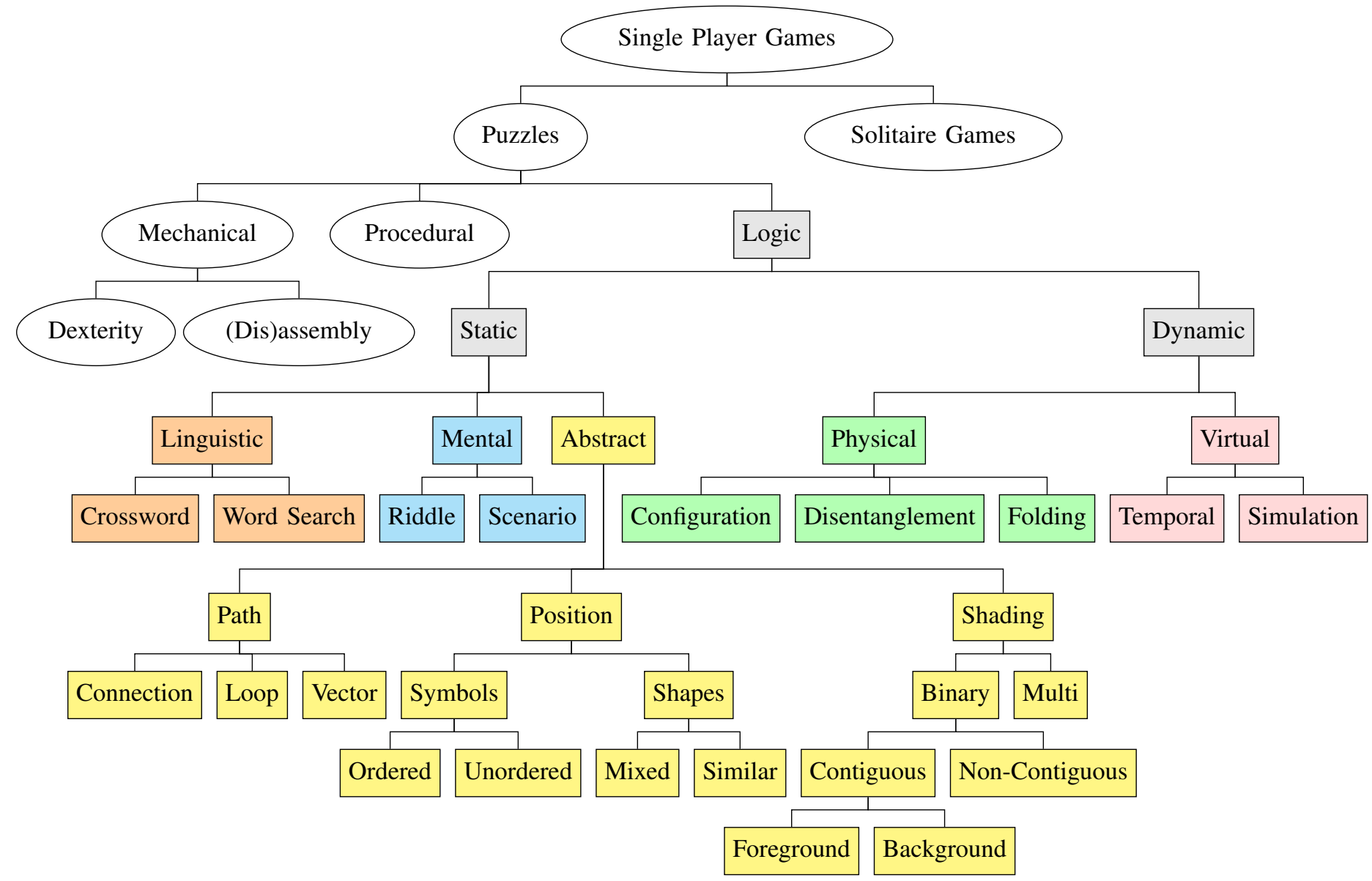

Fig. 2: Taxonomy of logic puzzles within the broader context of single player games.

between puzzles and solitaire games by observing that puzzles have definite solutions while solitaire games are not guaranteed to have a solution, e.g. in solitaire card games such as Patience there is a random element as to whether a given challenge may be solved or not.

In our taxonomy, the main distinction is between Static and Dynamic types of logic puzzles, as defined below. We colour their subcategories in the figure to help group them visually.

\section{A. Static Logic Puzzles}

Static logic puzzles start in an incomplete state and require the solver to complete the challenge to reach a solution state, through the monotonic accumulation of information deduced from prior states. They can typically be played using pen and paper. We identify the following subcategories:

1) Abstract: Abstract logic puzzles are languageindependent and provide the solver with a set of options that need to be instantiated to a single solution value according to the rules. They tend to use basic shapes, symbols - including numbers and characters - and geometric primitives. Abstract logic puzzles are the focus of this paper.

2) Linguistic: Linguistic logic puzzles often closely resemble Abstract puzzles in appearance and function, but depend on the language in which they are presented. Linguistic puzzle challenges (Section VII) do not tend to translate well, as doing so can require changing the challenges themselves to suit the new language.

3) Mental: Mental logic puzzles are lateral thinking exercises that rely more heavily on cognitive processes. Unlike Linguistic puzzles, they can be more easily translated as the thought process behind their solution is language-independent, even though they are framed using sentences, questions or statements.

\section{B. Dynamic Logic Puzzles}

Dynamic logic puzzles change states in a nonmonotonic fashion and may involve cycles in play. These puzzles may involve two goal states, in which the aim is progress from one to the other, and back again, ad infinitum. They include:

1) Physical: These come as physical objects, which the solver manipulates to reach a specified goal state.

2) Virtual: Digital modelling allows mechanisms not possible in the other logic puzzle subcategories, such as incorporating time or recreating puzzles from elsewhere in the hierarchy.

In this paper, we are primarily concerned with the Abstract logic puzzles category, which covers the Nikoli-style puzzles that we want to model. The following section describes this category in more detail. The other subcategories within our 


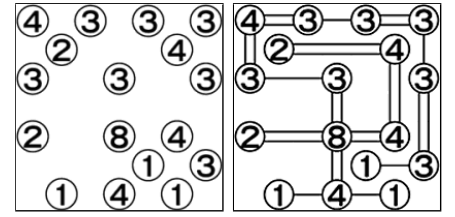

(a) Hashi

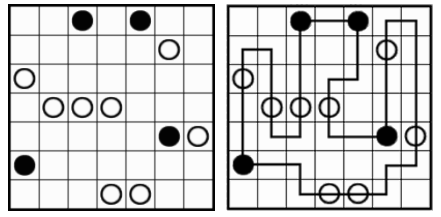

(b) Masyu

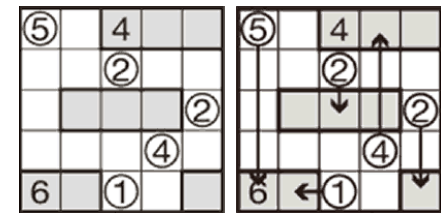

(c) Yosenabe

Fig. 3: Examples from the Path category.

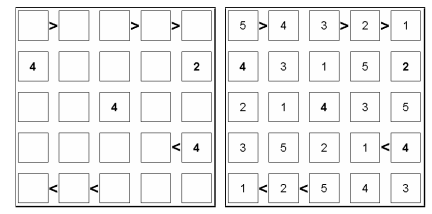

(a) Futoshiki

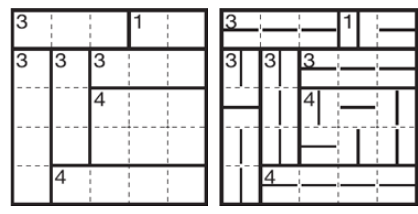

(b) Juosan
Fig. 4: Examples from the Position/Symbols category.

taxonomy (Fig. 2) will be described in more detail in a full report to be produced as part of this study.

\section{Abstract Logic Puzzles}

This section describes the various subcategories of Abstract logic puzzles in more detail.

\section{A. Path}

The solver aims to complete one or more paths by drawing lines or curves. This includes:

1) Connection: The solver aims to complete connections between specified elements (typically cells or vertices), with or without loops and/or crossings. Fig. 3a shows an example. This is the simplest form of Path puzzles.

2) Loop: Closing the path into a loop is the aim in itself. Fig. $3 \mathrm{~b}$ shows an example. This does not include puzzles in which loops form as a side-effect.

3) Vector: The solver draws arrows to indicate direction and occupy space as part of the solution process. Fig. 3c shows an example. If the arrows must form loops, then the puzzle belongs to the Loop class instead.

\section{B. Position}

The solution is based on the position of puzzle elements placed by the solver. This includes:

1) Symbols: Some puzzle elements - typically cells or vertices - contain symbols, such as numbers or characters. This can include lines or other geometric shapes.

Puzzles of this type are described as Ordered if the symbols have ordinal relationship and their order is relevant to the solution, otherwise they are described as Unordered. Figs. 4a and $4 \mathrm{~b}$ show Ordered and Unordered Symbols examples, respectively.

2) Shapes: A shape is a grouping of one or more orthogonal cells. Typically, the solver is required to draw a border around the shapes, dividing the Active Field (Section VII) between them. This class of puzzles is least likely to feature pre-set elements (Section VII).

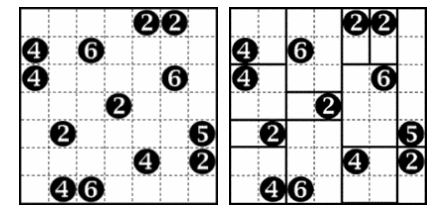

(a) Shikaku

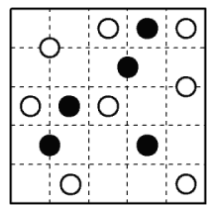

(b) Tentai Show

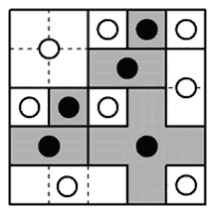

Fig. 5: Examples from the Position/Shapes category.

Shapes might be restricted to be Similar, or they might be Mixed to allow a variety of shapes. Figs. $5 \mathrm{a}$ and $5 \mathrm{~b}$ show Similar and Mixed examples, respectively.

\section{Shading}

Shading logic puzzles involve shading cells to achieve the solution. They work with cell 'colour', such as:

1) white (or empty),

2) black (or darkly shaded), and

3) grey (or lightly shaded).

Colours are only counted as such when they fill the whole cell; cells cannot be partially coloured or reliant on orientation. The hints for these puzzles can often be given in the Frame of the challenge rather than in its Active Field (Section VII]).

The solver has to colour the challenge cells, which may start out empty or contain hints, according to the rules. If the puzzle features symbols and/or lines, in addition to colours, then their respective categories take precedence over the Shading category. We identify the following subcategories:

1) Binary: Cells can be shaded either of two possible colours, usually white or black. This is the most common class of Shading puzzles. Same-coloured cells can be arranged contiguously (Contiguous) with either a focus on white (Background) or black (Foreground $]_{4}^{4}$ The alternative is the NonContiguous case, depending on the puzzles' rules. Fig. 6a and $6 \mathrm{~b}$ show Background and Foreground Binary examples, respectively, while Fig. 7 a shows a Non-Contiguous Binary challenge.

2) Multi: Multi-colour puzzles involve solutions with more than two colours. Orthogonal cells must typically be different colours. Fig. $7 \mathrm{~b}$ shows a Multi example.

\section{Discussion}

Our taxonomy covers all cases encountered in our corpus of 100 logic puzzles, including puzzles that mix goals from

\footnotetext{
${ }^{4}$ Here, we use the convention that the background is white and the foreground markings are black, as per pen \& paper versions of these puzzles.
} 


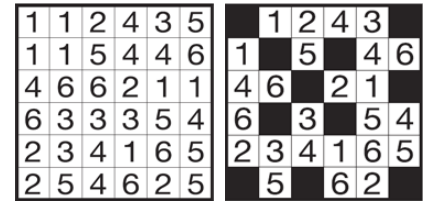

(a) Hitori

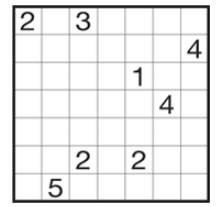

(b) Nurikabe

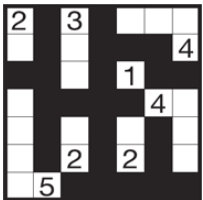

Fig. 6: Examples from the Shading/Binary/Contiguous category.

different parts of the hierarchy. Examples include Yajilin (draw a loop and colour leftover cells) and Pencils (draw pencil shapes and then draw lines through leftover cells). However, we observe that in such mixed-goal cases, one goal tends to dominate and is typically the defining characteristic of the puzzle. In addition, the approaches to solving such mixed-goal cases more closely resemble the approaches used for puzzles from the dominant category. We therefore classify such cases by their dominant goal aspect, e.g. Yajilin is a Path type and Pencils is a Position/Shape/Mixed type.

We have also encountered ambiguous cases such as Shakashaka (draw black triangles that form white rectangular shapes) and Juosan (draw a horizontal or vertical line in every cell, Fig. 4b). These were resolved within our taxonomy by specifying the criteria for their respective categories more precisely; as a result, a prioritisation arises where more specific categories take precedence over less specific ones. For example, Loop takes precedence over Vector and the Placement classes take precedence over any class in the Shading category. Thus, Shakashaka is a Similar Shapes puzzle (instead of NonContiguous) and Juosan is an Unordered Symbols puzzle (instead of Connection).

Our hierarchy effectively subdivides the range of Japanese logic puzzles into useful categories, based on both the puzzle mechanisms and their goals. The solution approaches for the various puzzle types are generally more alike the closer they are within our taxonomy, which helps our ultimate goal of implementing the full range of puzzles.

\section{CONCLUSION}

We have classified 100 puzzles using our taxonomy. Few ambiguities were observed, which are resolved by the prioritisation rules of the conflicting (sub)classes. Preliminary results show that puzzles within each class share similar rules and require similar approaches to solving. We will now apply our taxonomy to the task of implementing the full range of pen $\&$ paper, or Japanese logic puzzles, as part of a larger project.

\section{ACKNOWLEDGEMENT}

This research is part of the ERC-funded Digital Ludeme Project (Consolidator Grant \#771292) being run by Cameron Browne at Maastricht University. Puzzle images Figs. 3c, 4b. 5b, 6a and 6b are from [4]. Figs. 1, 3a, 3b, 5a and 7a are from [2], Fig. 4a is from https://en.wikipedia.org/wiki/Futoshiki and Fig. $7 b$ is from [5].

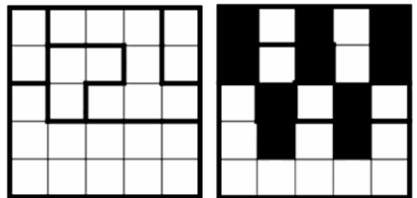

(a) Norinori

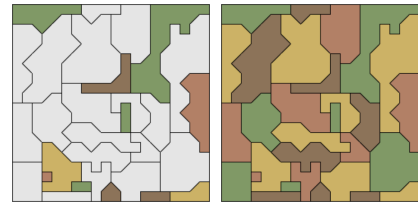

(b) Map
Fig. 7: Examples from the Shading/Binary/Non-contiguous and Shading/Multi categories.

\section{REFERENCES}

[1] T. Times, Japanese Logic Puzzles: Hitori, Hashi, Slitherlink and Mosaic. Harper Collins, 2006.

[2] A. Janko and O. Janko. (1999) Nikoli puzzle encyclopedia. [Online]. Available: https://www.janko.at/Raetsel/Nikoli/index-2.htm

[3] Nikoli. (2001) Nikoli's puzzles (japanese). [Online]. Available: https: //www.nikoli.co.jp/ja/puzzles/

[4] -. (2001) Nikoli's puzzles (english). [Online]. Available: https: //www.nikoli.co.jp/en/puzzles/index.html

[5] S. Tatham. (2019) Simon tatham's portable puzzle collection. [Online]. Available: https://www.chiark.greenend.org.uk/ sgtatham/puzzles/

[6] S. Kutasov and I. Morozov. (1998) Cross+a. [Online]. Available: http://www.cross-plus-a.com/puzzles.htm

[7] M. R. Johnson, "Casual games before casual games: Historicizing paper puzzle games in an era of digital play," Games and Culture, vol. 14, no. 2, pp. 119-138, 2019.

[8] A. Janko and O. Janko. (1999) Rules. [Online]. Available: https: //www.janko.at/Raetsel/Rules.htm

[9] C. Browne, "The nature of puzzles," Game \& Puzzle Design, vol. 1, no. 1, pp. 23-34, 2015.

\section{APPENDIX A. GlossARY}

This glossary of terms used throughout the paper is partially derived from Janko's list of common phrases [8]:

Active Field The set of all cells that are either set or unset.

Challenge An instance of a puzzle, as used in [9].

Frame The space around the Active Field that is still part of the puzzle. It can be empty or contain hint information.

Pre-set element Elements, such as cells or edges, that come in a pre-set state and cannot be changed.

Puzzle Field The entirety of the puzzle, including both the active field and the frame.

Region A group of orthogonally connected cells surrounded by a border.

(Un)set elements A puzzle element (i.e. cell, edge or vertex) is unset while it has a range of possible values. It becomes set when it is instantiated to a single solution value. 